\title{
Complete Endoscopic Closure (Clipping) of a Large Esophageal Perforation After Pneumatic Dilation in a Patient with Achalasia
}

\author{
Sergio Coda, MD, PhD, ,2 Fabio Antonellis, MD, , Sokratis Tsagkaropulos, MD, \\ Federico Francioni, $\mathrm{MD}^{3}$ and Paolo Trentino, $\mathrm{MD}^{2}$
}

\begin{abstract}
The risk of esophageal perforation following endoscopic balloon dilation for achalasia is in the range of $1 \%-5 \%$, with a mortality rate of $1 \%-20 \%$. Perforations need to be recognized early, and, if reasonable, an immediate endoscopic repair should be pursued quickly. Herein, we report a case of successful endoscopic closure by clipping of a large iatrogenic perforation in a patient with achalasia. An 80-year-old woman with achalasia was admitted to our institution to undergo pneumatic dilation. A 40-mm balloon dilator with inflation pressure of 20 psi was used for 2 minutes as usual. During the procedure, the patient had a transient bradycardia. Endoscopic control showed a 2-cm rupture of the distal esophagus. Prompt endoscopic repair of the perforation by endoclips $(n=6)$ was then attempted, followed by conservative management by total parenteral nutrition and intravenous antibiotics. Endoscopic clipping completely closed the esophageal perforation. The patient was given oral nutrition 10 days later without any complications. Six months after discharge from the hospital, the patient was healthy and free of dysphagia. Endoscopy showed complete healing of the esophageal mucosa without luminal stenosis. This report highlights that prompt endoscopic clipping is a useful means to close a large esophageal perforation caused by pneumatic dilation.
\end{abstract}

\section{Introduction}

$\mathbf{T}$ HERAPEUTIC DILATION for achalasia requires the forceful distension of the lower esophageal sphincter to partially disrupt the circular muscle and lastingly lower its pressure. The major complication of endoscopic balloon dilation (EBD) is esophageal perforation with an incidence between $1 \%$ and $5 \%$. When perforations occur, the mortality rate can reach as high as $20 \%$ and correlates with delays in both diagnosis and treatment. ${ }^{1}$

If any substantial perforation has occurred, an immediate endoscopic repair should be attempted. Herein, a case of successful endoscopic closure by clipping of a large iatrogenic perforation immediately after EBD in a patient with achalasia is reported.

\section{Case Report}

An 80-year-old woman with a long history of achalasia attended our institution to undergo endoscopic pneumatic dilation on an outpatient basis as usual. She was given only liquid food for 2 days before the dilation as per normal local practice. The procedure was conducted with the patient under conscious sedation and continuous pulse oximetry monitoring.

The placement of the dilator was performed under fluoroscopic guidance. A 40-mm achalasia balloon dilator (Rigiflex ${ }^{\circledR}$; Boston Scientific, Boston, MA) with an inflation pressure of $20 \mathrm{psi}(1034 \mathrm{~mm} \mathrm{Hg})$ for 2 minutes was used as per standard practice. During the procedure, the patient had a transient bradycardia. Immediate postdilation inspection showed a 2-cm rupture of the distal esophagus immediately above the cardia. Prompt endoscopic repair of the tear by apposition of endoclips (Resolution ${ }^{\mathrm{TM}}$ clips; Boston Scientific) was then attempted.

Clips were placed first at the margins of the rupture to prevent any further widening and afterward in a centripetal direction. The complete closure required a total of six clips. Unfortunately, no image capture device was connected at the

\footnotetext{
${ }^{1}$ Endoscopy Unit, Department of Gastroenterology, Imperial College London, London, United Kingdom.

${ }^{2}$ Endoscopy Unit and ${ }^{3}$ Division of Thoracic Surgery, Department of Surgery "Paride Stefanini," "Sapienza" University of Rome, Rome, Italy.
} 


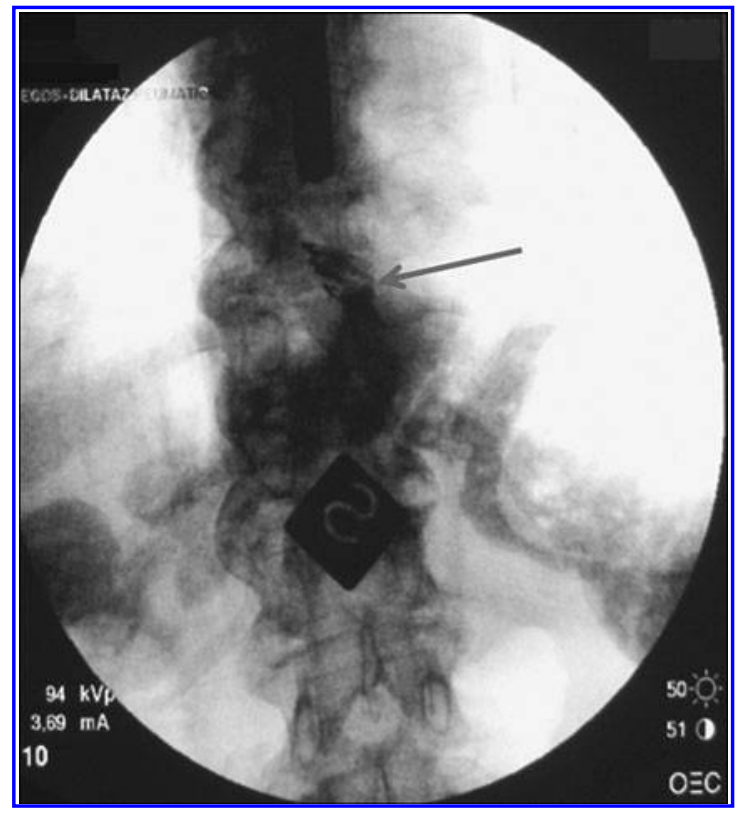

FIG. 1. Fluoroscopy showing the clips attached (arrow) to close the esophageal perforation immediately after the rupture repair.

time of endoscopy so a fluoroscopic image was taken to document the event (Fig. 1). Conservative management consisting of total parenteral nutrition, intravenous antibiotics, and clinical observation followed.

One hour after the rupture repair, a chest computed tomography scan showed the presence of pneumomediastinum, minimal bilateral pleural effusion, and no periesophageal fluid or extraluminal contrast (Fig. 2). Endoscopic clipping closed completely the esophageal perforation as the esophagoscopy control confirmed the day after (Fig. 3).

One week later, sodium amidotrizoate/meglumine amidotrizoate (Gastrografin ${ }^{\circledR}$; Bayer Schering Pharma AG, Berlin, Germany) swallow and functional magnetic resonance imaging assessed the complete transit of the contrast bolus to the stomach (Fig. 4).

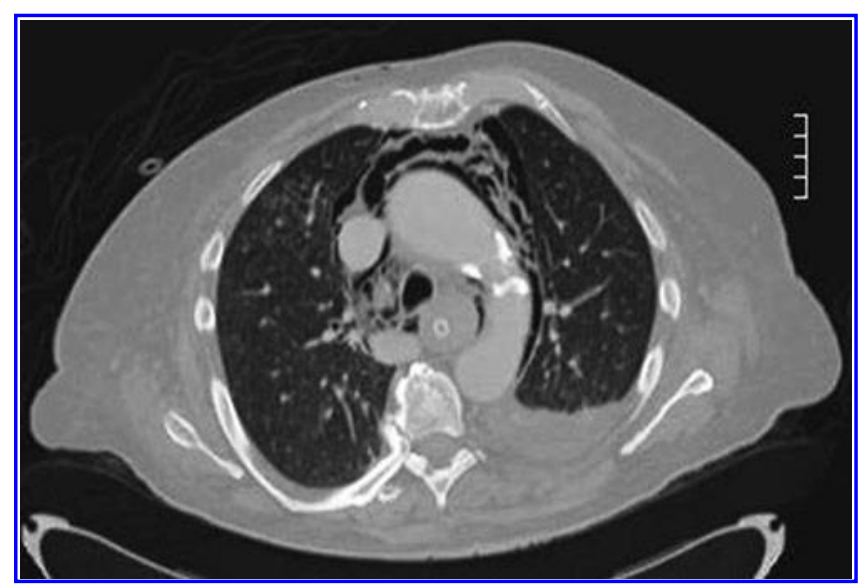

FIG. 2. One hour after the rupture repair, chest computed tomography scan shows pneumomediastinum, with minimal bilateral pleural effusion and no periesophageal fluid or extraluminal contrast.

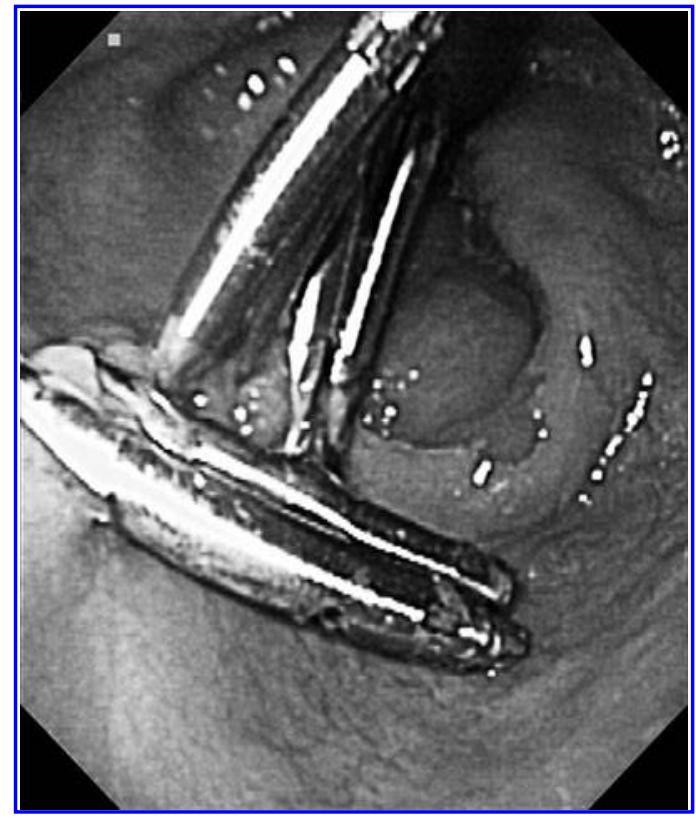

FIG. 3. Endoscopic view of closed perforation 1 day after the rupture repair.

The patient was given oral nutrition 10 days after the perforation without any complication. One day before discharge from the hospital (Day 15), the computed tomography scan confirmed complete resolution of the pneumomediastinum and absence of lung densities or pleural effusion.

Six months later, the patient was healthy and did not complain of dysphagia or other dysmotility symptoms. Endoscopy showed complete healing of the esophageal mucosa. None of the clips remained in place, and no stenosis of the esophageal lumen resulted.

\section{Discussion}

Pneumatic dilation of the lower esophageal sphincter is still the first-line therapy for the treatment of achalasia, while laparoscopic Heller myotomy with partial fundoplication (Dor or Toupet) is typically reserved for patients who have persistent dysphagia after multiple dilatations or who have suffered a perforation during EBD. ${ }^{2}$ Although less effective than EBD in the long term, botulinum toxin intrasphincteric injection is another viable endoscopic option in young patients, patients not responsive to pneumatic dilation, or patients not able or not willing to undergo surgery. In this case, botulinum toxin intrasphincteric injection was, perhaps improperly, not considered a viable option as the patient had no significant co-morbidities and had always responded well to EBD in the past. Also, the balloon was, perhaps, not adequately sized, given the long history of achalasia and repeated dilations. Outcome expectations and risks of each therapeutic option over time should be carefully considered and weighed in the decision-making process. Peroral endoscopic myotomy has recently been indicated as an alternative treatment to surgery, although it is currently used in the context of research only, and its clinical value is still under investigation. ${ }^{3}$

Acute perforations are potentially life-threatening emergencies in which prompt closure is required to avoid 


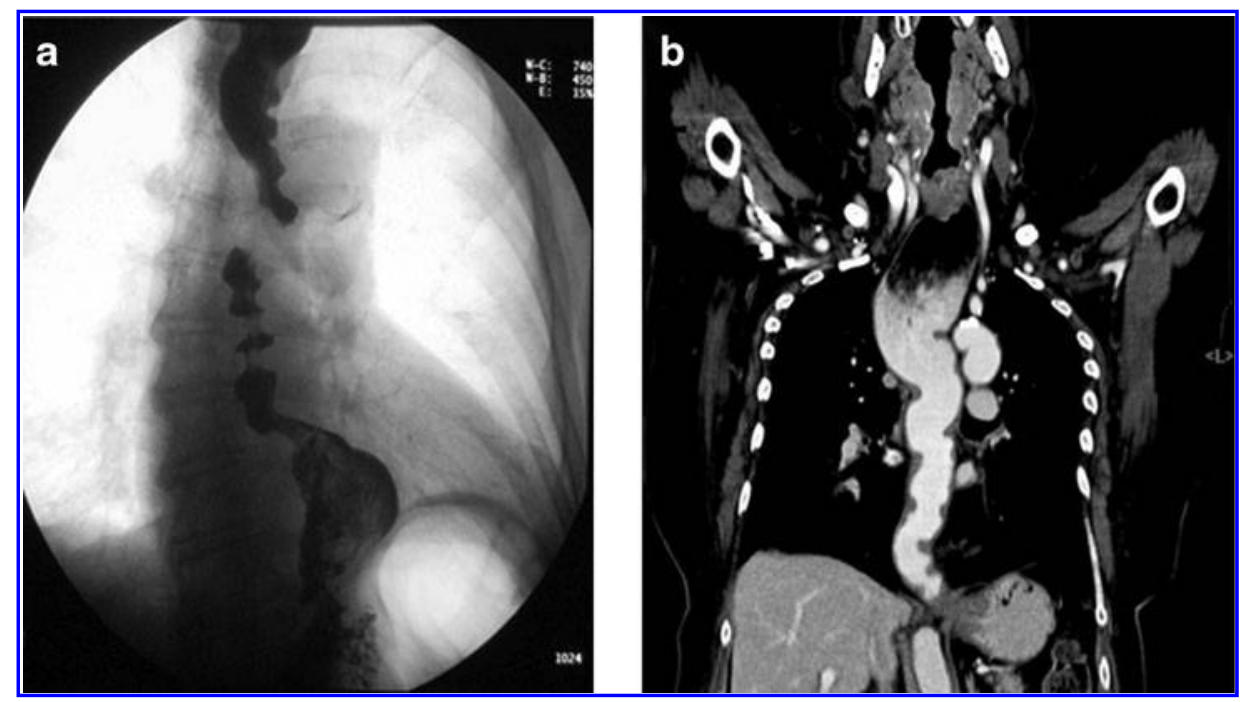

FIG. 4. One week after the rupture repair, (a) Gastrografin swallow roentgenogram and (b) functional magnetic resonance imaging show complete transit of the contrast bolus to the stomach.

contamination of visceral spaces. For many years surgery has represented the only adequate treatment, especially in patients with a significant contamination. Alternatively, temporary esophageal stenting has been recently used in the treatment of patients with iatrogenic perforations with immediate and complete sealing of the ruptures. ${ }^{4}$ Conservative approach has also been reported without the need for surgery, especially for nontransmural tears. ${ }^{5}$

Only a few cases of iatrogenic esophageal perforation in patients with achalasia and managed by endoscopic clipping have been reported in the English language literature. As with our case, the authors clipped the tear immediately after recognizing the complication. ${ }^{6,7}$ Pooled analysis for demographic and perforation variables over a period of 40 years, along with predictors for closure time after clipping, identified only the duration of perforation as a significant predictor of closure time. ${ }^{8}$ In a prospective 7-year follow-up study on 32 patients with idiopathic achalasia, endoscope-guided pneumatic dilation proved safe and fairly effective, with only one perforation occurring and $61.7 \%$ cumulative clinical remission at the 7th year of follow-up; older patients ( $>45$ years) had a better overall outcome. ${ }^{9}$

This report shows that clipping is a useful means to close a large acute esophageal perforation caused by EBD and to prevent possible leakage of fluids in the mediastinum with a high risk of infection. It should be emphasized that the successful treatment of this case can be at least partially attributed to the increased confidence and expertise in the management of acute perforations as a result of the advanced training on endoscopic submucosal dissection recently pursued by one of the authors (S.C.) at one of the most experienced Japanese institutions. $^{10}$

Because of the lack of large prospective trials, the efficacy of clipping and the role of contamination, for large as well as for small timely repaired perforations, remain to be further determined. Provided that the complication is promptly recognized and that the edges of the tear are clean and easily approachable, clipping should be considered as a safe and useful technique to attempt to obliterate even large iatrogenic esophageal perforations. Surgery should be indicated only in cases where a breakdown of the repair site occurs. However, caution, close observation of worsening signs, and a tight cooperation with the thoracic surgeons, to guard against any further complications, are strongly recommended.

\section{Disclosure Statement}

No competing financial interests exist.

\section{References}

1. Brinster CJ, Singhal S, Lee L, Marshall MB, Kaiser LR, Kucharczuk JC. Evolving options in the management of esophageal perforation. Ann Thorac Surg 2004;77:14751483.

2. Boeckxstaens GE, Annese V, des Varannes SB, et al. Pneumatic dilation versus laparoscopic Heller's myotomy for idiopathic achalasia. N Engl J Med 2011;364:1807-1816.

3. Inoue $\mathrm{H}$, Minami $\mathrm{H}$, Kobayashi $\mathrm{Y}$, et al. Peroral endoscopic myotomy (POEM) for esophageal achalasia. Endoscopy 2010;42:265-271.

4. van Heel NCM, Haringsma J, Spaander MCW, Bruno MJ, Kuipers EJ. Short-term esophageal stenting in the management of benign perforations. Am J Gastroenterol 2010; 105:1515-1520.

5. Vanuytsel T, Lerut T, Coosemans W, et al. Conservative management of esophageal perforations during pneumatic dilation for idiopathic esophageal achalasia. Clin Gastroenterol Hepatol 2012;10:142-149.

6. Wehrmann T, Stergiou N, Vogel B, Riphaus A, Kockerling F, Frenz MB. Endoscopic debridement of paraesophageal, mediastinal abscesses: A prospective case series. Gastrointest Endosc 2005;62:344-349.

7. Martinek J, Kovacova S, Nosek V, Verner T, Vasicek M, Spicak J. Successful endoscopic treatment (clipping) of esophageal perforation during balloon dilatation in a patient with achalasia. Endoscopy 2008;40(Suppl 2):E61-E62.

8. Qadeer MA, Dumot JA, Vargo JJ, Lopez AR, Rice TW. Endoscopic clips for closing esophageal perforations: Case 
report and pooled analysis. Gastrointest Endosc 2007;66: 605-611.

9. Chuah S-K, Hu T-H, Wu K-L, et al. Clinical remission in endoscope-guided pneumatic dilation for the treatment of esophageal achalasia: 7-year follow-up results of a prospective investigation. J Gastrointest Surg 2009;13: 862-867.

10. Coda S, Trentino P, Antonellis F, et al. A Western singlecenter experience with endoscopic submucosal dissection for early gastrointestinal cancers. Gastric Cancer 2010;13: 258-263.
Address correspondence to: Sergio Coda, $M D, P h D$ Department of Medicine Imperial College London

Room 731, Blackett Laboratory South Kensington Campus Prince Consort Road London SW7 2AZ United Kingdom

E-mail: s.coda@imperial.ac.uk 
